\title{
Modelling quasicrystals at positive temperature
}

\author{
Hans Koch ${ }^{1}$ and Charles Radin ${ }^{1,2}$
}

\begin{abstract}
We consider a two-dimensional lattice model of equilibrium statistical mechanics, using nearest neighbor interactions based on the matching conditions for an aperiodic set of 16 Wang tiles. This model has uncountably many ground state configurations, all of which are nonperiodic. The question addressed in this paper is whether nonperiodicity persists at low but positive temperature. We present arguments, mostly numerical, that this is indeed the case. In particular, we define an appropriate order parameter, prove that it is identically zero at high temperatures, and show by Monte Carlo simulation that it is nonzero at low temperatures.
\end{abstract}

\section{Introduction}

Certain alloys are believed to exhibit, at low temperature, a state of thermal equilibrium which is solid but not crystalline (as determined for instance by X-ray diffraction), a state called quasicrystalline [1].

In part to explain the observed diffraction patterns, it is common to model the energy ground state of such a material by using (aperiodic) tilings [1, 2, 3]. But ever since such models have been proposed [4,5] there has been the need to determine whether they actually are useful to explain the behavior of materials at positive temperature, that is, it is unclear whether such models exhibit a phase transition from the usual disorder at high temperature to an aperiodically ordered state at low but positive temperature.

We will analyze a two-dimensional lattice "tiling" model with appropriate energy ground states in an attempt to make some progress in this problem. The lattice model is of a standard form [6, 7]. In fact the specific model has been discussed already, by Leuzzi and Parisi [8], though they concentrated on the degeneracy of the energy ground state of the model, and a possible connection with the nonequilibrium behavior of glasses and similar materials. See also [9].

We will not be giving a proof of a phase transition in this model. Indeed, there are very few models for which one can prove phase transitions. A proof of a quasicrystalline phase may well require a new basic technique. But at least it would be useful to have a good order parameter, and to develop some intuition about the nature of the order, on which to base a future argument for a transition. This is our goal. We will introduce such an order parameter for our model, prove that it vanishes identically at high temperature, and present numerical evidence that it is nonzero below some critical value of the temperature.

\footnotetext{
1 Department of Mathematics, The University of Texas at Austin, Austin, TX 78712

2 Research supported in part by NSF Grant DMS-0700120
} 
relative weight $\exp [-\beta H(\sigma)]$ to a tile configuration $\sigma$, where $H(\sigma)$ is the number of defects of $\sigma$. The minimizers of $H$, also referred to as ground state configurations, are precisely the $A$-tilings. (It is not difficult to prove that any state obtained as a limit $\beta \rightarrow \infty$ gives full measure to these configurations $[11,12]$.)

To test for long range order, we only consider the type $(S$ or $L)$ of the four tile edges. This identification by type defines an equivalence relation " $\sim$ " on $A$, with four equivalence classes. Given a configuration $\sigma$, an $A$-tiling $\gamma$, and a finite region $\Lambda \subset \mathbb{Z}^{2}$, denote by $\phi_{\Lambda}(\sigma, \gamma)$ the fraction of sites $j \in \Lambda$ where $\sigma_{j} \sim \gamma_{j}$. The limit as $\Lambda \uparrow \mathbb{Z}^{2}$, if it exists, will be denoted by $\bar{\phi}(\sigma, \gamma)$, and we define $\bar{\psi}(\sigma)=\int_{\mathcal{G}} \bar{\phi}(\sigma, \gamma) d \lambda(\gamma)$. One of our goals is to study the quantity

$$
Q_{\beta}(\gamma)=\int_{\mathcal{S}} \frac{\bar{\phi}(\sigma, \gamma)}{\bar{\psi}(\sigma)} d \nu_{\beta}(\sigma), \quad \gamma \in \mathcal{G}
$$

which measures how much a typical tile configuration at inverse temperature $\beta$ aligns with the ground state configuration $\gamma$. In the absence of any preference, $Q_{\beta}$ is the constant function 1 , or equivalently, the "order parameter"

$$
q(\beta)=\int_{\mathcal{G}} Q_{\beta}(\gamma) \ln \left[Q_{\beta}(\gamma)\right] d \lambda(\gamma)
$$

is identically zero. We will prove that this is the case for sufficiently small $\beta>0$. This result is independent of the choice of boundary condition in defining $\nu_{\beta}$ through the energy function $H$.

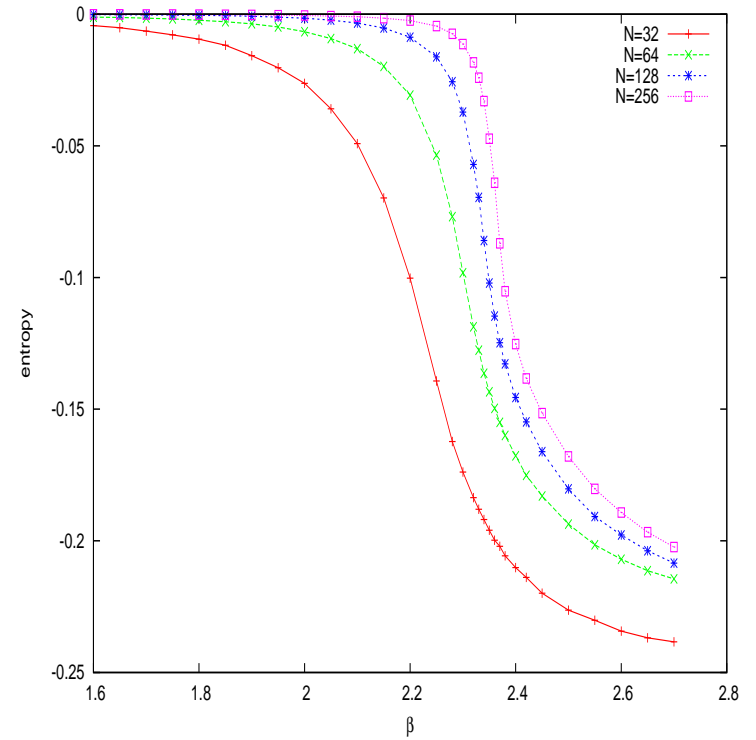

Fig. 2 The order parameter $q_{\beta}$.

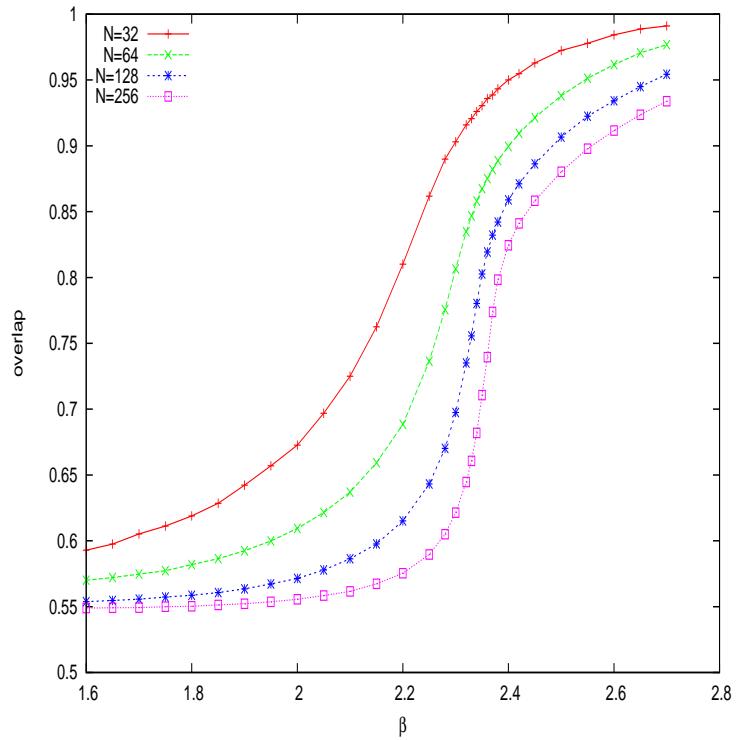

Fig. 3 The overlap $Q_{\beta}(\tau)$.

Our remaining results are purely numerical. In what follows, we choose as boundary conditions (at infinity) a fixed $A$-tiling $\tau$. It is important to keep this in mind. Fig. 2 shows the values of $q(\beta)$ obtained via Monte Carlo simulations, for tile configurations of 
size $N \times N$, with $N$, a power of 2 , ranging from 32 to 256 . These results suggest that $q(\beta)$ becomes negative as $\beta$ is increased past a critical value $\beta_{c} \approx 2.4$. If correct, this would imply the existence of a phase transition, from a disordered state for $\beta<\beta_{c}$ to an ordered state for $\beta>\beta_{c}$, where translation invariance is broken. As one would expect, $Q_{\beta}(\gamma)$ takes its maximum at $\gamma=\tau$. Fig. 3 shows the simulated values of $Q_{\beta}(\tau)$.

Similar signs of an order-disorder transition were found in [8], using Monte Carlo simulations for $8 \leq N \leq 32$, with free (but eventually frozen) boundary conditions. At these values of $N$, there is evidence that the phase transition is of second order, with a power-law or logarithmic divergence of the specific heat (as $\beta \rightarrow \beta_{c}$ ), depending on the model used to fit the data. Our numerical results for $32 \leq N \leq 256$ clearly favor the second alternative, if either. In fact, we find a slowdown in the increase of the specific heat, suggesting that the phase transition is of third or higher order. Our simulated values for the energy per tile, and for the specific heat, are shown in Figs. 4 and 5 . The curve labeled $\mathrm{dE} / \mathrm{dT}$ is the (discrete) derivative of the energy, for $N=256$, and the other three curves in Fig. 5 were obtained from the energy fluctuations.

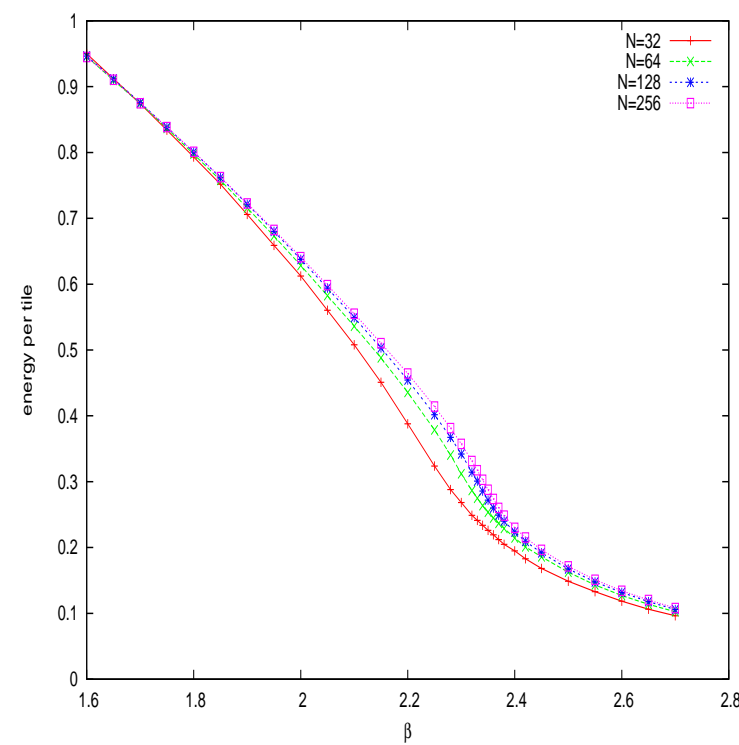

Fig. 4 The average energy per tile.

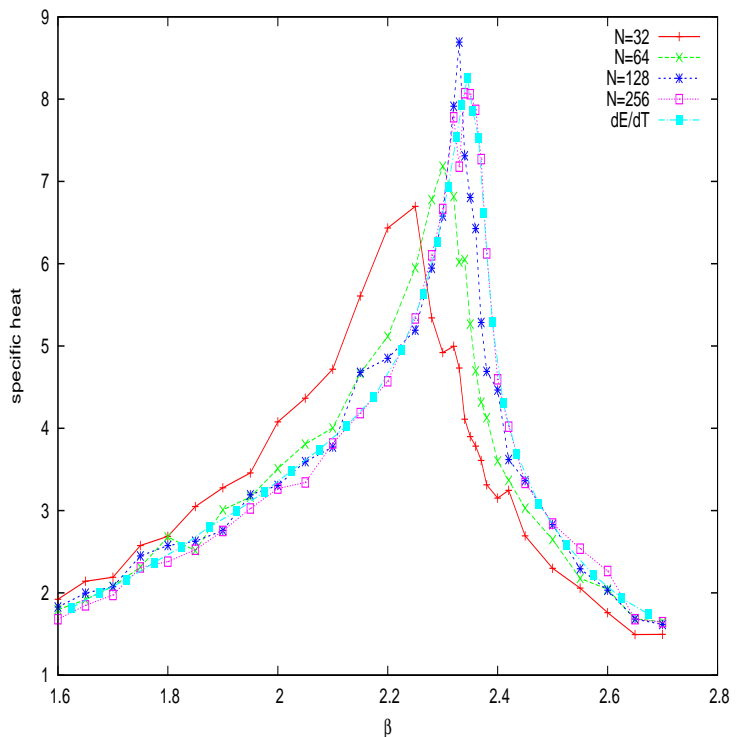

Fig. 5 The specific heat.

Fig. 6 shows a two-point correlation $C_{\beta}(d)$ as a function of the (vertical) separation $d$. For $\beta>\beta_{c}$, the correlation approach a nonzero constant that depends on $\beta$, indicating again the existence of long range order at low temperature. The correlation length $C_{\beta}^{-1}(1 / 4)$ is shown in Fig. 7. It diverges roughly like $\left(\beta_{c}-\beta\right)^{-7}$ as $\beta \uparrow \beta_{c}$. Such power law behavior is again similar to what is observed in models with ordered low temperature phases, except that the exponent 7 is unusually large. By contrast, the correlations for $\beta \approx \beta_{c}$ decay roughly like $\exp \left(-c d^{1 / 2}\right)$, at least in the observed range. This suggests that there is no renormalization fixed point (nontrivial scaling limit) associated with the critical value $\beta_{c}$. Instead, there seems to be a "line of fixed points" for $\beta>\beta_{c}$. This is a feature that is better known in models with a continuous (internal) symmetry, such as the $X Y$-model. 


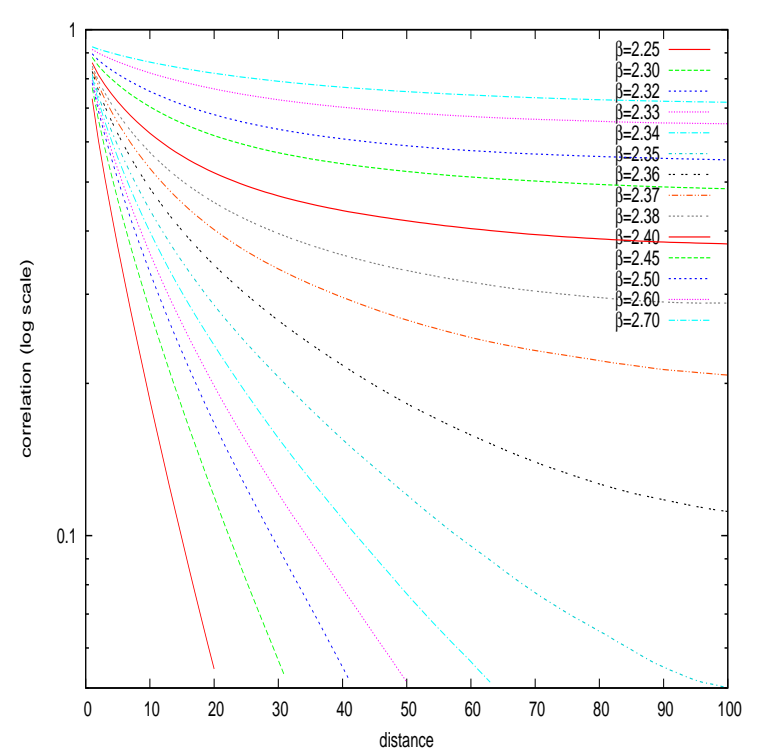

Fig. 6 Correlation: $C_{\beta}(d)$ versus $d$.

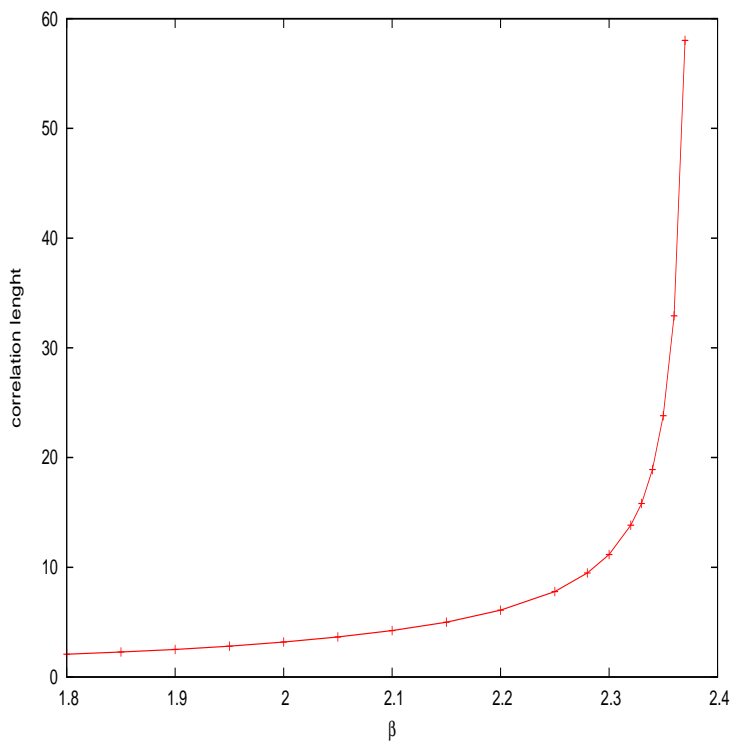

Fig. 7 Correlation length $C_{\beta}^{-1}(1 / 4)$.

\subsection{Further observations}

We now give a description of the ground states, that can be used to compute and visualize the function $Q_{\beta}$. It should also provide some insight into the behavior of the model at low temperature.

Without the normalizing factor $\bar{\psi}^{-1}$ in the integral (2.1), our function $Q_{\beta}$ is analogous to the order parameters used in models that have periodic ground states and/or a compact internal symmetry group. By being a function on $\mathcal{G}$, it is implicitly covariant under any symmetry of the model, including translations. If none of the symmetries are broken, then the corresponding "entropy" $q(\beta)$ vanishes. Thus, $Q_{\beta}$ seems to be a natural order parameter in models with a large number of ground states. For the tiling model considered here, this number is uncountable. Nevertheless, $Q_{\beta}$ is easy to compute. The reason is that, as we will see below, $\mathcal{G} / \sim$ can be identified with the torus $\mathbb{T}^{2}$, where translations $\left(T^{j} \sigma\right)_{i}=\sigma_{i+j}$ act by irrational rotations. Each equivalence class $[a]=\{x \in A: x \sim a\}$ of $A$ corresponds to one of four disjoint rectangles in a covering of $\mathbb{T}^{2}$, and $\psi_{\Lambda}^{-1}(\sigma) \phi_{\Lambda}(\sigma, \gamma)$ is the probability that the rectangle $\left[\sigma_{j}\right]$ contains the point $T^{j} \gamma$, for a randomly chosen site $j \in \Lambda$.

To be more precise, given any $X, Y \in\{S, L\}$, denote by $X \times Y$ the set (equivalence class) of all prototiles in $A$ whose horizontal and vertical edges are of type $X$ and $Y$, respectively. Let $R=\{S \times S, S \times L, L \times S, L \times L\}$. Then to every tile configuration $\sigma: \mathbb{Z}^{2} \rightarrow A$ we can associate a function $[\sigma]: \mathbb{Z}^{2} \rightarrow R$, by setting $[\sigma]_{j}=\left[\sigma_{j}\right]$. If $\gamma$ is an $A$-tiling, then $[\gamma]$ is a product of two Fibonacci sequences $k \mapsto x_{k}$ and $k \mapsto y_{k}$, in the sense that $[\gamma]_{j}=x_{j_{1}} \times y_{j_{2}}$. Conversely, any product of two Fibonacci sequences can be obtained in this way. Thus, the set of all such $R$-tilings $[\gamma]$ is the product $\mathcal{F} \times \mathcal{F}$, where $\mathcal{F}$ denotes the set of all Fibonacci sequences. Since $Q_{\beta}(\gamma)$ only depends on the equivalence class $[\gamma]$, it suffices to find a convenient description for the Fibonacci sequences. (In fact, $[\gamma]=\{\gamma\}$ for almost every tiling $\gamma$, but we will not use this here.) 
One such description is the following [2, p. 128]. Given a real number $\vartheta$, and a partition $\{J(S), J(L)\}$ of the circle $\mathbb{T}=\mathbb{R} / \mathbb{Z}$, we can associate with any angle $\alpha \in \mathbb{T}$ a sequence $x \in\{S, L\}^{\mathbb{Z}}$ by setting

$$
x_{k}= \begin{cases}S, & \text { if } \alpha+k \vartheta \in J(S) \\ L, & \text { if } \alpha+k \vartheta \in J(L) .\end{cases}
$$

Let now $\vartheta$ be the inverse golden mean, $\vartheta=\frac{1}{2}(\sqrt{5}-1)$. The Fibonacci sequences are obtained by choosing either $J(L)=[0, \vartheta)$ and $J(S)=[\vartheta, 1)$, or else $J(L)=(0, \vartheta]$ and $J(S)=(\vartheta, 1]$. The two sets of sequences differ only by a countable set, corresponding to angles $\alpha+k \vartheta$ that are zero (modulo 1 ). This set has measure zero, so we can ignore it. Thus, for our purposes, $\mathcal{F}$ can be identified with the circle $\mathbb{T}$.

In this representation, translations $\left(T^{k} x\right)_{m}=x_{m+k}$ on $\mathcal{F}$ become irrational rotations $R^{k} \alpha=\alpha+k \vartheta$ on the circle. Similarly, $\mathbb{Z}^{2}$-translations on $\mathcal{F} \times \mathcal{F}$ are represented by irrational rotations $R^{j} \alpha=\left(\alpha_{1}+j_{1} \vartheta, \alpha_{2}+j_{2} \vartheta\right)$ on the torus $\mathbb{T}^{2}$. In both cases, the unique invariant measure is Lebesgue measure. We will describe later how these properties can be used for numerical computations.

While a ground state configuration $\gamma$ determines a point $\alpha$ on the torus, an $n \times n$ "patch" of $\gamma$ determines a rectangular neighborhood of $\alpha$ of linear size $\mathcal{O}\left(n^{-1}\right)$. Thus, a typical low temperature configuration determines locally a pair of "fuzzy" angles. Unlike in the $X Y$-model, the energy associated with a gradual change of the column (row) angle over a horizontal (vertical) distance $d$ does not decrease with $d$. This follows from the fact that the density of letter-mismatches between two Fibonacci sequences is asymptotically proportional to the angle difference. A similar argument may apply in the other directions, based on the "slanted" Fibonacci sequences described in the remark below. Thus, it seems plausible that the model can maintain long range order at low temperatures, despite the fact that there are uncountably many ground states.

By analogy with the $X Y$-model, one might ask about the existence of vortices and/or dipoles in our tiling model. The question is meaningful only at reasonably low temperature, since the angles are ill defined at high temperature. So isolated vortices are unlikely to play a major role. But in a model with slowly varying angles, a dipole can be associated with two successive crossings through a fixed value. Such dipoles (horizontal and vertical) are a prominent feature at temperatures near $\beta_{c}$. But they are rarely isolated and thus hard to analyze systematically. Besides these horizontal/vertical dipoles, one can also observe "slanted" dipoles whose ends are single defects (not type mismatches), and whose connecting line has a slope near $\vartheta^{ \pm 1}$. These slanted dipoles seem to be the main source of entropy at very low temperatures. Their density has no visible singularity over the range of temperatures considered, so they do not appear to play a major role in the observed phase transition.

Remark. In a different representation of the Ammann tilings [10], the 16 prototiles are rectangles, whose $L$-edges and $S$-edges have lengths $\vartheta$ and $1-\vartheta$, respectively. In this representation, it is possible to replace the numeric edge-labels by two types of line segments, say blue and green, transverse to the edges, such that a perfect tiling is characterized by the blue (and similarly the green) segments combining into a parallel sequence of straight lines, known as Ammann bars. The bars are slanted, with slopes $\vartheta^{ \pm 1}$, and it should not 
be too surprising that the spacings between the blue (as well as the green) bars define a Fibonacci sequence. The same can be done with square tiles, except that the bars are only piecewise linear. This shows that the matching rules for the Ammann tiles enforce, primarily and in a direct way, products of Fibonacci sequences.

\subsection{The model}

In this section, we give a more detailed description of the model and show that the order parameter (2.2) vanishes for small $\beta>0$. We recall that our simulations were carried out with boundary conditions given by a tiling $\tau$. For the purpose of this section, $\tau$ could be any configuration in $\mathcal{S}$. Thus, we shall suppress the dependence on $\tau$ in our notation.

We start by considering finite regions $\Lambda \subset \mathbb{Z}^{2}$. The energy $H_{\Lambda}(\sigma)$ of a configuration $\sigma \in \mathcal{S}$ is defined as the number of defects of $\sigma$ that intersect $\Lambda$. Given a real number $\beta>0$, and a finite subset $\Lambda$ of $\mathbb{Z}^{2}$, the Gibbs state (for $\Lambda$ ) at temperature $1 / \beta$, with boundary condition $\tau$, is the functional that assigns to a continuous function $f$ (for the product topology) on $\mathcal{S}$ the value

$$
\langle f\rangle_{\beta, \Lambda}=Z_{\beta, \Lambda}^{-1} \sum_{\sigma \in \mathcal{S}_{\Lambda}} f(\sigma) e^{-\beta H_{\Lambda}(\sigma)} .
$$

Here, $\mathcal{S}_{\Lambda}$ is the set of configurations $\sigma \in \mathcal{S}$ that agree with $\tau$ outside $\Lambda$, and $Z_{\beta, \Lambda}$ is a normalization constant, determined by the condition $\langle 1\rangle_{\beta, \Lambda}=1$. Taking a limit $\Lambda \uparrow \mathbb{Z}^{2}$ along squares defines a Gibbs measure $\nu_{\beta}$ on $\mathcal{S}$,

$$
\int_{\mathcal{S}} f d \nu_{\beta}=\langle f\rangle_{\beta} \stackrel{\text { def }}{=} \lim _{\Lambda \uparrow \mathbb{Z}^{2}}\langle f\rangle_{\beta, \Lambda} .
$$

By well known results in the theory of lattice models $[13,14]$, the measure $\nu_{\beta}$ for small positive $\beta$ is translation invariant, ergodic, and mixing. In particular, $\nu_{\beta}$ does not depend on the choice of boundary condition $\tau$. (For large $\beta$ the limit may have to be taken along subsequences, and it can depend on $\tau$.)

Let $\nu$ be any translation-invariant probability measure on $\mathcal{S}$, and consider the space $\Omega=\mathcal{S} \times \mathcal{G}$, equipped with the product measure $\mu=\nu \times \lambda$. This measure is invariant under translations $T^{j}(\sigma, \gamma)=\left(T^{j} \sigma, T^{j} \gamma\right)$. Thus by the ergodic theorem [15], if $\phi$ is any function in $\mathrm{L}^{1}(\Omega)$, then the orbit averages

$$
\phi_{n}(\sigma, \gamma)=\frac{1}{4 n^{2}} \sum_{j_{1}=-n}^{n-1} \sum_{j_{2}=-n}^{n-1} \phi\left(T^{j}(\sigma, \gamma)\right)
$$

converge $\mu$-almost everywhere to a function $\bar{\phi}$ in $\mathrm{L}^{1}(\Omega)$, as $n \rightarrow \infty$. In what follows, let $\phi(\sigma, \gamma)=\theta\left(\sigma_{0} \sim \gamma_{0}\right)$, where $\theta$ (true) $=1$ and $\theta$ (false $)=0$. Now assume that $\nu$ is mixing. Then, by a standard result in ergodic theory [16, p. 228], the measure $\mu$ is ergodic. As a result, $\bar{\phi}$ is the constant function with value $\int_{\Omega} \phi d \mu$.

This shows that the function $Q_{\beta}$ defined in (2.1) is well defined, as long as the Gibbs measure $\nu_{\beta}$ is translation invariant. Furthermore, if $\nu_{\beta}$ is mixing, then this function is identically 1 , and $q(\beta)=0$. As mentioned above, this holds for sufficiently small $\beta>0$. 
We note that these arguments do not show that $Q_{\beta}$ is well defined for all values of $\beta$, although this seems likely to be true. (It is true for instance at zero temperature, as we will see later.) In any case, if translation invariance is broken at some $\beta>0$, then a phase transition has to occur.

The correlations described earlier are given by

$$
\Gamma_{\beta}(i)=\left\langle V_{0} V_{i}\right\rangle_{\beta}-\left\langle V_{0}\right\rangle_{\beta}\left\langle V_{i}\right\rangle_{\beta}, \quad i \in \mathbb{Z}^{2}
$$

where $V_{i}=V \circ T^{i}$, and where $V: \mathcal{S} \rightarrow \mathbb{R}$ is defined as follows. Denote by $x_{j}(\sigma)$ the horizontal type $\left(L\right.$ or $S$ ) of the tile $\sigma_{j}$ in a configuration $\sigma$. Define $f(S)=-1$ and $f(L)=1$. Then $V(\sigma)$ is the value of $f\left(x_{j}(\sigma)\right)$, averaged over all sites $j$ in some fixed finite region containing the origin. The data in Fig. 6 are only for the correlation $C_{\beta}(d)=\Gamma_{\beta}(i)$ in the vertical direction $i=(0, d)$. The correlations for $V$ in other directions have been computed as well, but they are not shown here. They have large oscillations (related to the Fibonacci sequence) and are less convenient for estimating a correlation length. We also considered a purely probabilistic measure of correlations, namely the relative entropy of the random variable $(j, \sigma) \mapsto x_{j}(\sigma)$ and its translates. The results are not qualitatively different from those found via (2.7).

\section{Computations}

Using the correspondence between ground states (modulo equivalence) and points on the torus, the overlap (2.6) of a configuration $\sigma$ with a tiling $\gamma$ can now be written as follows. Let $\alpha$ be the point on $\mathbb{T}^{2}$ defined by $\gamma$. Writing $\left[\sigma_{j}\right]=x_{j} \times y_{j}$, we have

$$
\phi_{n}(\sigma, \gamma)=\frac{1}{4 n^{2}} \sum_{j_{1}=-n}^{n-1} \sum_{j_{2}=-n}^{n-1} \chi\left(J\left(x_{j}\right), \alpha_{1}+j_{1} \vartheta\right) \chi\left(J\left(y_{j}\right), \alpha_{2}+j_{2} \vartheta\right)
$$

where $b \mapsto \chi(B, b)$ denotes the indicator function of a set $B \subset \mathbb{T}$. The integral of $\phi_{n}$ over $\alpha \in \mathbb{T}^{2}$ is given by

$$
\psi_{n}(\sigma)=\frac{1}{4 n^{2}} \sum_{j_{1}=-n}^{n-1} \sum_{j_{2}=-n}^{n-1}\left|J\left(x_{j}\right)\right|\left|J\left(y_{j}\right)\right| .
$$

As was shown earlier, $\psi_{n}^{-1} \phi_{n} \rightarrow 1$ as $n \rightarrow \infty$, for small $\beta$. The limit can also be computed at zero temperature. In this case, the double sum in (3.1) factorizes into a product of simple sums. Without loss of generality (due to translation invariance), we can assume that $\sigma$ corresponds to the torus point 0. By using the ergodicity of irrational rotations, one finds that $\phi_{n} \rightarrow \bar{\phi}$ a.e. on $\mathcal{G} \times \mathcal{G}$, with $\bar{\phi}(\sigma, \gamma)=\varphi\left(\alpha_{1}\right) \varphi\left(\alpha_{2}\right)$, where $\varphi$ is the piecewise linear function

$$
\varphi(t)= \begin{cases}1-2|t|, & \text { if }|t|<1-\vartheta \\ 2 \vartheta-1, & \text { otherwise }\end{cases}
$$

The functions $\psi_{n}$ converge a.e. to the constant $\kappa^{2}$, where $\kappa=\vartheta^{2}+(1-\vartheta)^{2}$. 
We expect that $Q_{\beta}(\gamma) \approx \kappa^{-2} \varphi\left(\alpha_{1}\right) \varphi\left(\alpha_{2}\right)$ for large values of $\beta$. This is indeed observed numerically, but this is to be expected in a finite system. For comparison, we show in Fig. 8 the computed values of $Q_{\beta}(\gamma)$, as a function of the point $\alpha \in \mathbb{T}^{2}$, for the inverse temperatures 2.3 and 2.4. (The observed transition for $N=256$ is between these values.)

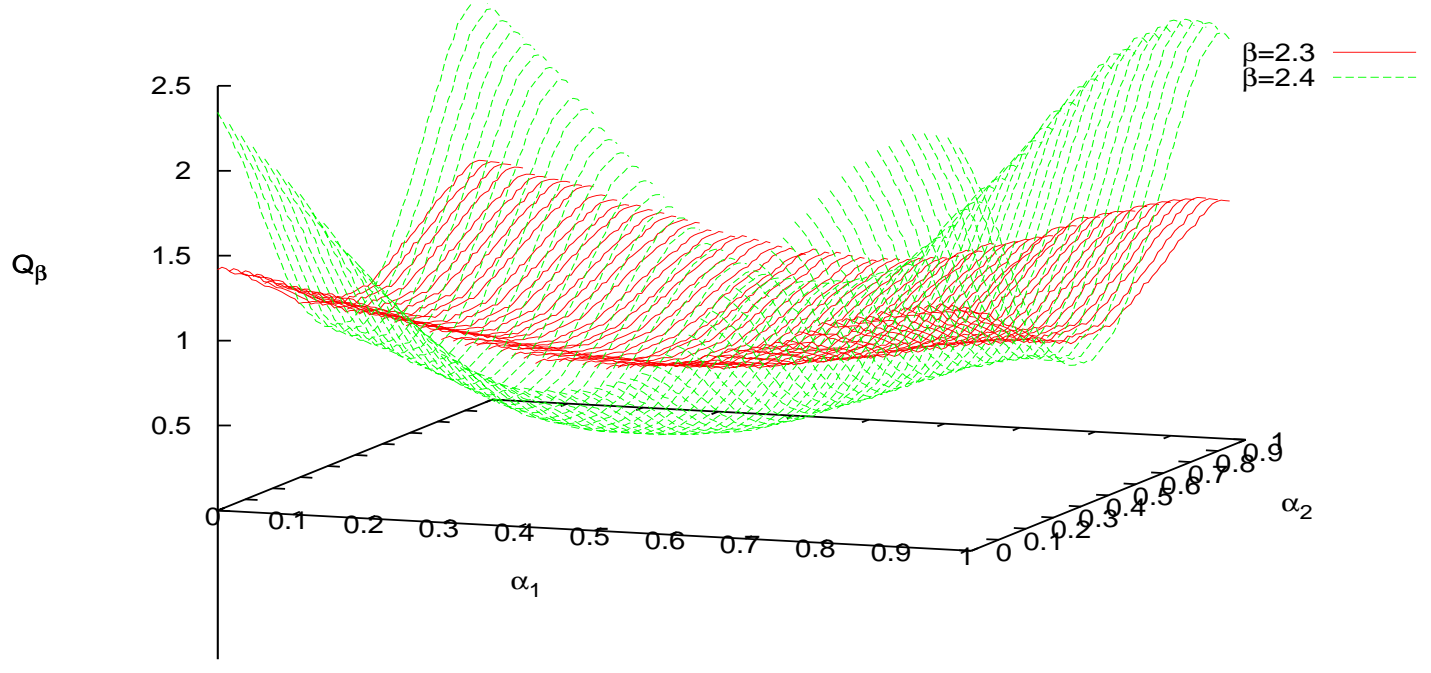

Fig. 8 Overlap $Q_{\beta}$ with the different ground states.

We recall that our simulations were carried out with boundary conditions given by a tiling $\tau$. This is why (and where) the graphs in Fig. 8 have a single maximum. If we had used other boundary conditions, then the limit of $\sigma_{\beta}$ as $\beta \rightarrow \infty$ would be a mixture of pure tiling states, possibly very complicated, and the system might resemble a spin glass, as was observed for instance in [8]. In this context, we should mention that the number of defect-free configurations on an $N \times N$ lattice square is bounded by $e^{c N}$, as was already described in [8]. The number of such configurations that can be extended to a full $A$-tiling is only $\mathcal{O}\left(N^{2}\right)$.

In our numerical computations, we evaluate the sum in (3.1) on a finite $256 \times 256$ grid of points $\alpha \in \mathbb{T}^{2}$. (Choosing a finer grid gives no significant improvement.) Averaging $\phi_{n}$ over $\alpha$ then yields $\psi_{n}$. This is done for each individual tile configuration $\sigma$ in a collection $\Sigma_{2 n, \beta}$, obtained via Monte Carlo simulation. Then we perform the integral (2.1) by averaging $\psi_{n}^{-1} \phi_{n}$ over the configurations in $\Sigma_{2 n, \beta}$.

Each of our ensembles $\Sigma_{N, \beta}$ contains $10^{3}$ configurations. For increased flexibility, they were computed beforehand and stored for analysis later [17]. The configurations in $\Sigma_{N, \beta}$ are separated from each other by at least $M_{N, \beta}$ Monte-Carlo steps (updates of individual tiles), where $M_{N, \beta}$ was determined by monitoring overlaps with the appropriate starting configuration, and settling times for various observables, to eliminate any visible dependence or bias. To give an example, $M_{256,2.45} \approx 2.3 * 10^{12}$. The initial configurations 
for $N=256$ were obtained by slowly cooling a random configuration. Patches of the resulting configurations were also used to generate the starting points for $N<256$.

\section{References}

[1] C. Janot, Quasicrystals: A Primer, Oxford University Press (1997).

[2] M. Senechal, Quasicrystals and geometry, Cambridge University Press (1995).

[3] C. Radin, Miles of Tiles, American Mathematical Society (1999).

[4] A. Mackay, Crystallography and the Penrose pattern, Physica 114A, 609-613 (1982).

[5] D. Levine, P.J. Steinhardt, Quasicrystals: a new class of ordered structures, Phys. Rev. Lett. 53, 2477-2480 (1984).

[6] D. Ruelle, Thermodynamic Formalism, Addison-Wesley (1978).

[7] C. Radin, Tiling, periodicity, and crystals, J. Math. Phys. 26, 1342-1344 (1985).

[8] L. Leuzzi, G. Parisi, Thermodynamics of a tiling model, J. Phys. A 33, 4215-4225 (2000).

[9] J. Miȩkisz, Many phases in systems without periodic ground states, Commun. Math. Phys. 107, 577-586 (1986).

[10] B. Grünbaum, G.C. Shephard, Tilings and Patterns, Freeman (1986).

[11] D. Ruelle, Some remarks on the ground state of infinite systems in statistical mechanics, Commun. Math. Phys. 11, 339-345 (1969).

[12] R. Schrader, Ground states in classical lattice systems with hard core, Commun. Math. Phys. 16, 247-264 (1970).

[13] D. Ruelle, Statistical mechanics, rigorous results, W.A. Benjamin Inc. (1969).

[14] B. Simon, The statistical mechanics of lattice gases, Volume 1, Princeton University Press (1993).

[15] A. Nevo, Pointwise ergodic theorems for actions of groups, in Handbook of Dynamical Systems, IB, ed. A. Katok, B. Hasselblatt, Elsevier (2005).

[16] I. Cornfeld, S. Fomin, Ya. Sinai, Ergodic Theory, Springer-Verlag (1982).

[17] The numerical ensembles $\Sigma_{N, \beta}$ are available from the authors upon request. 